\title{
Numerical study of roughness and contact angle effects on water transport in a gas channel
}

\author{
Alex Jarauta ${ }^{a}$, Thomas Chan $^{b}$, Adam Weber ${ }^{b}$, Pavel Ryzhakov, Jordi Pons-Prats \\ and Marc Secanell ${ }^{a}$
}

\author{
${ }^{a}$ Energy Systems Design Laboratory, Department of Mechanical Engineering \\ University of Alberta \\ Edmonton AB T6G 1H9, Canada \\ e-mail: secanell@ualberta.ca,web page: http://www.esdlab.mece.ualberta.ca/ \\ ${ }^{b}$ Energy Conversion Group \\ Lawrence Berkeley National Laboratory \\ 1 Cyclotron Road MS 70R108B \\ Berkeley CA 94720, US
}

\begin{abstract}
Water accumulation in fuel cell anode and cathode channels can lead to hydrogen starvation and therefore, severe electrode degradation, and significantly fuel cell performance deterioration. For this reason, liquid water transport in micro-channels remains an active area of research in fuel cells as well as many other research areas [1,2]. Two-phase flow in micro-channels is governed by surface tension and viscous effects and involves the interaction of air, water and the solid substrate. To date most numerical studies have been performed using volume of fluid (VOF) [3] or level set (LS) [4] implementations in commercial software, however these methods are usually explicit thereby limiting the maximum time step that can be used. An investigation on new methods to solve two-phase flows in micro-channels is key to develop alternative methods that allow for faster simulation time and allow to study physical process that remain a challenge, such as interface conditions between the channel and porous media in fuel cells [5].

In this work, a novel formulation based on a Lagrangian-Eulerian formulation is presented and experimentally validated [6]. The governing equations for both air and water are the NavierStokes equations. Air is represented using a fixed mesh, whereas a moving mesh is used to discretize the water domain. This formulation is particularly advantageous to the problem at hand, since it allows for exact tracking the air-water interface. An implicit term is used to represent the surface tension effects, allowing us to use time steps greater than those from explicit formulations [7].

For the validation of the model, several experiments have been performed in a transparent microchannel. Droplet deformation and shedding on three substrates, i.e, Kapton, PTFE and a gas diffusion layer (Toray H60 10\% PTFE), is studied both numerically and experimentally. The goal of the experiments is to reproduce different conditions for injected water in a microchannel. Kapton and PTFE are smooth surfaces, the former being hydrophilic and the latter hydrophobic, whereas the GDL is a hydrophobic rough substrate and is used to emulate the conditions in a fuel cell channel. Two cameras are used to capture the emergence of water into the channel. The first camera is used to obtain images of the droplet's deformation from a lateral point of view, allowing us to measure the advancing and receding contact angles. The second camera obtains images along the channel, and therefore deformation effects of the droplet on the direction perpendicular to the airflow can also be quantified.
\end{abstract}

The presented model can predict droplet emergence, deformation and posterior detachment. Numerical results are consistent with the experimental data. For instance, the advancing contact angle remains approximately constant in rough surfaces, whereas the receding contact angle decreases, showing a slight increase prior to droplet's detachment. Results obtained with the current model are also compared to VOF results previously reported in literature and large discrepancies with the evolution of droplet deformation are observed. The model is shown to be 
able to predict the conditions that lead to droplet, slug and film flow in fuel cell channels.

\section{REFERENCES}

[1] M. Wörner. Numerical modeling of multiphase flows in microfluidics and micro process engineering: a review of methods and applications. Microfluid Nanofluid, 12:841-886, 2012.

[2] R. B. Ferreira, D. S. Falcão, V. B. Oliveira, and A. M. F. R. Pinto. Numerical simulations of two-phase flow in proton exchange membrane fuel cells using the volume of fluid method - A review. Journal of Power Sources, 277:329-342, 2015.

[3] X. Zhu, P.C. Sui, and N. Djilali. Numerical simulation of emergence of a water droplet from a pore into a microchannel gas stream. Microfluid Nanofluid, 4:543-555, 2008.

[4] N. Akhtar, A. Qureshi, J. Scholta, C. Hartnig, M. Messerschmidt, and W. Lehnert. Investigation of water droplet kinetics and optimization of channel geometry for PEM fuel cell cathodes. International Journal of Hydrogen Energy, 34:3104-3111, 2009.

[5] A. Z. Weber, R. L. Borup, R. M. Darling, P. K. Das, T. J. Dursch, W. Gu, D. Harvey, A. Kusoglu, S. Litster, M. M. Mench, R. Mukundan, J. P. Owejan, J. G Pharoah, M. Secanell, and I. V. Zenyuk. A critical review of modeling transport phenomena in Polymer-Electrolyte fuel cells. Journal of the Electrochemical Society, 161(12):F1254-F1299, 2014.

[6] A. Jarauta, P. B. Ryzhakov, M. Secanell, P. R. Waghmare, and J. Pons-Prats. Numerical study of droplet dynamics in a polymer electrolyte fuel cell gas channel using an embedded Eulerian-Lagrangian approach. Journal of Power Sources, 323:201-212, 2016.

[7] M. Sussman and M. Ohta. A stable and efficient method for treating surface tension in incompressible two-phase flow. SIAM Journal on Scientific Computing, 31:2447-2471, 2009. 